\title{
Detection of Unhealthy Plant Leaves using Image Processing with Arduino
}

\author{
Kirupa Ganapathy, O. Manikanta Sreevatsa
}

\begin{abstract}
India is a cultivating country and as for seventy percentage of our people depends upon agribusiness. Third of our national compensation starts from agribusiness. Along these lines the illness disclosure of plants expects a critical activity inside the agricultural field. Larger piece of the plant contaminations are realized by the attack of bacterium, parasites, disease, etc. In the occasion that correct consideration isn't taken during this space, it should cause real impacts on plants and unfairly impacts the productivity and quality. To perceive, the plant ailments we'd like a snappy modified techniques. The most approach grasped in seek after for area and unmistakable verification of plant contaminations is eye observation through stars. the basic leadership limit of AN informed conjointly depends upon his/her adequacy, like exhaustion and vision, work weight air, etc subsequently this technique is time outstanding and more expensive. Here an endeavor is masterminded with a thought of police work plant diseases abuse picture process. Picture methodology instrument chest of Matlab is used for evaluating affected space of infection and to see the capability inside the shade of the illness impacted space. This idea may be loosened up to find the reactions of any variety of plant ailments that is affected on absolutely remarkable horticulture crops. The algorithmic rule may be wont to arrange the leaves and the gathered outcomes are detached abuse Arduino basically based vehicle system This decreases an essential task of watching of farms crops at starting period itself to find the sign of sicknesses show up on plant leaves.
\end{abstract}

Keywords: Contamination in plants, Picture methodology, Segmentation.

\section{INTRODUCTION}

India is standard for horticultural nation; whereby about $70 \%$ of the populace relies upon farming. Ranchers have wide choice of variety to choose suitable yields for their homestead. Nonetheless, the development of harvests as ideal outcome and its nature or quality turn out will be commonly specialized. It will be optimized by the assistance of innovative help. The administration of lasting yields needs shut prevailing especially for the administration of ailments which will affect creation extensively and afterwards the post-reap life. The picture procedure is best system utilized in agrarian applications for following capacities. Foresee ailment from picture of plants. The malady diagnosing is confined by human visual abilities because of the majority of

Revised Manuscript Received on December 11, 2019

* Correspondence Author

Dr. Kirupa Ganapathy, Professor \& Head Department, ECE Department, Saveetha school of engineering, SIMTS, Chennai, India.

O. Manikanta Sreevatsa, UG scholar, ECE Department, Saveetha school of engineering Chennai, India. the essential side effects territory unit minuscule. This strategy is dreary, time overpowering. there's need for style framework that precisely recognizes, characterize and quantitatively identifies malady indications. If there should arise an occurrence of plant ailment the ailment is comprehended as any hindrance of customary physiological work of plants, producing trademark indications. a sign could be a reality coincidental a certain something and is found out as verification of its reality. ailment is brought about by microorganism that is any operator causing sickness. Sickness the board could be a troublesome errand. Primarily sicknesses region unit seen on the leaves on plants or stems of the plant. Exact measurement of those outwardly discovered maladies, bugs, attributes has not considered in any case inferable from the entanglement of visual examples .In the majority of the cases sicknesses zone unit seen on the leaves or stems of the plant. subsequently acknowledgment of plants, forgets about and checking the infections, side effects of the sickness assault, assumes an essential job in undefeated development of yields. Consequently building up a pc vision framework to watch, perceive, and characterize ailment influenced on yields which can stay away from human impedance and in this way because précised fair call concerning ailment disease and its any valuation. The occasion of a programmed framework conjointly encourage ranchers abstain from counseling divine. Programmed discovery of leaf illnesses is most fundamental examination subject since it may demonstrate gain in watching mammoth fields of harvests, thus precisely watch the ailments from the side effects that blessing on the plant leaves. This permits machine vision that is to supply picture basically based here picture procedure assumes vital Role. The framework gives the ability to Capture picture, technique it and acquire result through picture process. In the globe, ranchers outwardly survey of harvests like organic products, vegetables and in this manner the like stricken by the different sickness for acknowledgment and arrangement. In ongoing writing, the picture procedure strategies region unit being wide and quickly utilized in horticultural field for sickness location and grouping. Tuker and Chakraborty,(2008) have gave programming bundle that distinguishes, portrayed and determined rates of leaf space pathologic abuse advanced picture process. Al-Bashish etal. (2011) built up a brisk and right strategy inside which the leaf maladies zone unit recognized and characterized with the help of k-implies division and neural systems based order. Programmed grouping of leaf ailments depends on high goals multispectral and stereo pictures (Bauer et al., 2011).Sugar beet leaves region unit utilized in this methodology. 


\section{LITERATURE SURVEY}

In Shanmugam Agasta Adline A. L Aishwarya N Krithika G talked about computerized sicknesses location utilizing remote detecting pictures [1-3]. Agriculturists are confronting misfortune because of different yield ailments. It ends up dreary to the cultivators to screen the harvests normally when the developed region is giganStic (in sections of land). The most huge piece of our examination is early identification the malady when it starts spreading on the top layer of the leaves utilizing remote detecting pictures [4]. This methodology has two stages: first stage manages preparing of solid and just as unhealthy datasets i.e.) the extraction of edge esteems from the picture, second stage manages observing of harvests and ID of specific illness utilizing vigilant edge location calculation and histogram examination and furthermore private the agriculturists with an early alarm message right away [5-6].

In Roberto A. Lotufo, Jennifer Campbell, G. Bruce Pike talked about cores are not legitimately unmistakable on traditional anatomical attractive reverberation pictures (MRI), it is conceivable to watch contrasts between the cores utilizing dissemination tensor imaging (DTI), as a result of their particular fiber direction. This work shows a technique to section the different cores of human thalamus utilizing dispersion MRI [7]. Our methodology is to utilize the watershed change and different ideas from scientific morphology to portion the cores. Be that as it may, to portion structures utilizing the tensor information delivered with DTI (rather than scalar pictures) the idea of a tensorial morphological angle (TMG) should be presented [8]. In light of the TMG, division of the cores of the thalamus was effective utilizing the watershed change. Our division is reliable with a histological map book. Since the proposed technique, rather than most of the DTI-based division strategies, doesn't require manual seed as well as surface situation, its outcomes are profoundly repeatable [9-10].

InBirBhanu, Jing Peng. This paper introduces a general way to deal with picture division and article acknowledgment that can adjust the picture division calculation parameters to the changing natural conditions [11]. Division parameters are spoken to by a group of summed up stochastic learning automata and got the hang of utilizing connectionist support learning procedures [12-14]. The edge-outskirt occurrence measure is first utilized as fortification for division assessment to diminish computational costs related with model coordinating during the beginning period of adjustment. This measure alone, notwithstanding, can not dependably foresee the result of article acknowledgment. Along these lines, it is utilized related to show coordinating where the coordinating certainty is utilized as a support sign to give ideal division assessment in a shut circle object acknowledgment framework [15]. The adjustment switches back and forth among worldwide and nearby division forms so as to accomplish ideal acknowledgment execution [16-18]. Results are displayed for both indoor and outside shading pictures where the exhibition improvement after some time is appeared for both picture division and item acknowledgment.

InHarshalWaghmare, RadhaKokare Plant infections cause major monetary and creation misfortunes just as decrease in both amount and nature of agrarian generation. Presently a day's, for managing enormous field of yields there is been expanded interest for plant leaf infection recognition framework. The basic issue here is to screen the wellbeing of the plants and identification of the particular infections. Studies demonstrate that the greater part of the plant ailment can be analyzed from the properties of the leaf. Hence leaf based sickness investigation for plants is an energizing new space. The system proposed for ID of plant ailment through the leaf surface examination and example acknowledgment. In this work we center around Grapes plant leaf sickness recognition framework. The framework takes a solitary leaf of a plant as an information and division is performed after foundation expulsion. The portioned leaf picture is then investigated through high pass channel to distinguish the ailing piece of the leaf. The fragmented leaf surface is recovered utilizing one of a kind fractal based surface element. Fractal based highlights are locally invariant in nature and in this manner gives a decent surface model. The surface of each autonomous infection will be unique. The separated surface example is then arranged utilizing multiclass SVM. The work arranges center around significant illnesses regularly saw in Grapes plant which are wool buildup and dark decay. The proposed methodology profits counsel of horticultural specialists effectively to ranchers with the precision of $96.6 \%$ [19].

Introduced a sort of reproducing human clever control technique for the cool stockpiling of products of the soil, dampness, thus an immediate effect on the qualityof the nourishment stockpiling of high-accuracy checking ecological parameters [20]. The framework accept the inserted chip as a center, through sensors, transmitters and $\mathrm{A} / \mathrm{D}$ converter for information securing, and understands the cool stockpiling condition parameter astute control by the product, which can successfully improve the nature of nourishment stockpiling, vitality protection essentially, the staggering expense.

\section{PROPOSED SYSTEM}

In example acknowledgment and in picture preparing, highlight extraction is a unique type of dimensionality decrease. At the point when the information to a calculation is too huge to ever be handled and it is suspected to be famously excess, at that point the information will be changed into a diminished portrayal set of highlights. fig1:Block diagram of our proposed model shown below

\section{MODULES:}

1. PREPROCESSING

2. SEGMENTATION

3. FEATURE EXTRACTION

4. CLASSIFICATION

5. MAX232 TTL

6. ARDUINO UNO

7. LCD 


\section{REFERENCES}

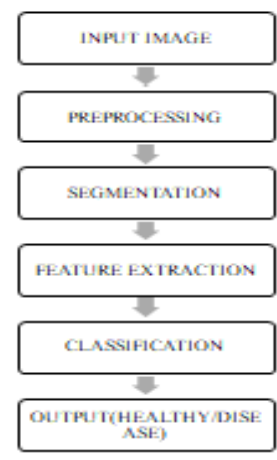

1. G. Patil, J. K., \& Kumar, R. (2011). "Advances in image processing for detection of plant diseases". Journal of Advanced Bioinformatics Applications and Research, 2(2), 135-141.

2. Revathi, P., and M. Hemalatha. "Classification of cotton leaf spot diseases using image processing edge detection techniques." 2012 International Conference on Emerging Trends in Science, Engineering and Technology (INCOSET). IEEE, 2012.

3. Al-Hiary, Heba, et al. "Fast and accurate detection and classification of plant diseases." International Journal of Computer Applications 17.1 (2011): 31-38.

4. Chaudhary, Piyush, et al. "Color transform based approach for disease spot detection on plant leaf." International Journal of Computer Science and Telecommunications 3.6 (2012): 65-70.

5. Arivazhagan, Sai, et al. "Detection of unhealthy region of plant leaves and classification of plant leaf diseases using texture features." Agricultural Engineering International: CIGR Journal 15.1 (2013): 211-217.

HARDWARE SECTION

6. Srivastava, Chanchal, et al. "Performance comparison of various filters and wavelet transform for image de-noising." IOSR Journal of Computer Engineering 10.1 (2013): 55-63.

7. Badnakhe, Mrunalini R., and Prashant R. Deshmukh. "Infected leaf analysis and comparison by Otsu threshold and k-means clustering." International Journal of Advanced Research in Computer Science and Software Engineering 2.3 (2012).

8. Al-Amri, Salem Saleh, Namdeo V. Kalyankar, and Santosh D. Khamitkar. "A comparative study of removal noise from remote sensing image." arXiv preprint arXiv:1002.1148 (2010).

Changing the information into the arrangement of highlights is called include extraction. In the event that the highlights separated are painstakingly picked it is normal that the highlights set will remove the pertinent data from the information so as to play out the ideal assignment utilizing this diminished portrayal rather than the full size info. Highlight extraction includes improving the measure of assets required to depict an enormous arrangement of information precisely. When performing investigation of complex information one of the serious issues comes from the quantity of factors included. Examination with an enormous number of factors for the most part requires a lot of memory and calculation control or a characterization calculation which over fits the preparation test and sums up ineffectively to new examples. Highlight extraction is a general term for techniques for building mixes of the factors to get around these issues while as yet portraying the information with adequate exactness.

\section{RESULT AND DISCUSSION}

Thus our objective in abstract is met. All kinds of leaves with different contamination level is captured and processed via image processing through Matlab. And all of the images with various contamination levels are processed with certain conditions using feature extraction. So for specific signal or if specific condition is attained (which is based on segmentation) arduino will display the level of contamination of the plant through the LCD Display.

\section{CONCLUSION}

\section{ADVANTAGES:}

- The sign of the RPSM is its strong coordinating plan, which considers both the geometric dissemination consistency and the textural closeness

- Constraint on the relative change is applied to keep from unreasonable face distorting.

\section{APPLICATION:}

- Used to archive and backup large image data set.

- Used to store data in database.

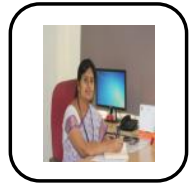

(anjay B., and Nitin P. Kumbhar. "Agricultural plant leaf disease detection using image processing." International Journal of Advanced Research in Electrical, Electronics and Instrumentation Engineering 2.1 (2013): 599-602.

10. Al Bashish, Dheeb, Malik Braik, and Sulieman Bani-Ahmad. "A framework for detection and classification of plant leaf and stem diseases." 2010 International conference on signal and image processing. IEEE, 2010

11. Ananthi, S., and S. Vishnu Varthini. "Detection and classification of plant leaf diseases." International Journal of research in engineering \& applied Sciences 2.2 (2012): 763-773.

12. Singh, Shalini S., and N. C. Chauhan. "K-means v/s K-medoids: A Comparative Study." National Conference on Recent Trends in Engineering \& Technology. Vol. 13. 2011.

13. Yerpude, Amit, and Sipi Dubey. "Colour image segmentation using K-medoids clustering." Int J Comput Technol Appl 3.1 (2012): 152-4.

14. Govindaraj.V, Sengottaiyan.G, - Survey of Image Denoising using Different Filters \|, IJSETR, 2013, 2(2), 344-351.

15. Tiger, Bindu, and Toran Verma. "Identification and classification of normal and infected apples using neural network." International Journal of Science and Research 2.3 (2013): 160-163.

16. Aji, Alham F., et al. "Detection of palm oil leaf disease with image processing and neural network classification on mobile device." International Journal of Computer Theory and Engineering 5.3 (2013): 528.

17. Gonzalez, Rafael C., and Richard E. Woods. "Digital image processing." (2002).

18. Beucher, Ottmar, and Michael Weeks. Introduction to MATLAB \& SIMULINK (A Project Approach). Laxmi Publications, Ltd., 2008.

19. Phiros Mansur, "Plant Leaf Recognition System Using Kernel Ensemble Approach", International Journal Of Advances In Signal And Image Sciences, Vol.4, No.1, 2018, pp. 30-36.

20. Murugan, S., and Srinivasan, C. "Underwater Object Recognition Using KNN Classifier", International Journal of MC Square Scientific Research, Vol. 9, No. 3, 2017, pp. 48-52.

\section{AUTHORS PROFILE}

Dr.KirupaGanapathy, Professor \& Head Department of Electronics and Communication Engineering, Saveetha Institute of Medical and Technical Sciences, Chennai, Tamilnadu, India

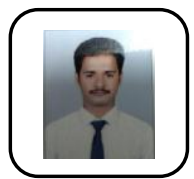

O.ManikantaSreevatsa Student, Department of ECE, Saveetha Institute of Medical and Technical Sciences, Chennai, Tamilnadu, India 